\title{
Developing a clinical translational neuroscience taxonomy for anxiety and mood disorder: protocol for the baseline- follow up Research domain criteria Anxiety and Depression ("RAD”) project
}

Leanne M. Williams ${ }^{1,2^{*}}$, Andrea N. Goldstein-Piekarski ${ }^{1,2+}$, Nowreen Chowdhry ${ }^{1,2+}$, Katherine A. Grisanzio ${ }^{1,2+}$, Nancy A. Haug ${ }^{1,3+}$, Zoe Samara ${ }^{1 \dagger}$, Amit Etkin ${ }^{1,2 \dagger}$, Ruth $\mathrm{O}^{\prime} \mathrm{Hara}^{1,2+}$, Alan F. Schatzberg ${ }^{1 \dagger}$, Trisha Suppes ${ }^{1,4 \dagger}$ and Jerome Yesavage ${ }^{1,2+}$

\begin{abstract}
Background: Understanding how brain circuit dysfunctions relate to specific symptoms offers promise for developing a brain-based taxonomy for classifying psychopathology, identifying targets for mechanistic studies and ultimately for quiding treatment choice. The goal of the Research Domain Criteria (RDoC) initiative of the National Institute of Mental Health is to accelerate the development of such neurobiological models of mental disorder independent of traditional diagnostic criteria. In our RDoC Anxiety and Depression ("RAD") project we focus trans-diagnostically on the spectrum of depression and anxiety psychopathology. Our aims are a) to use brain imaging to define cohesive dimensions defined by dysfunction of circuits involved in reactivity to and regulation of negatively valenced emotional stimulation and in cognitive control, b) to assess the relationships between these dimension and specific symptoms, behavioral performance and the real world capacity to function socially and at work and c) to assess the stability of brain-symptom-behavior-function relationships over time.

(Continued on next page)
\end{abstract}

\footnotetext{
* Correspondence: lea.williams@stanford.edu

${ }^{\dagger}$ Equal contributors

${ }^{1}$ Department of Psychiatry and Behavioral Sciences, Stanford University

School of Medicine, Stanford, CA 94305, USA

${ }^{2}$ Sierra-Pacific Mental IIIness Research, Education, and Clinical Center

(MIRECC) Veterans Affairs Palo Alto Health Care System, Palo Alto, CA 94304

USA

Full list of author information is available at the end of the article
} 
(Continued from previous page)

Methods and design: Here we present the protocol for the "RAD" project, one of the first RDoC studies to use brain circuit functioning to define new dimensions of psychopathology. The RAD project follows baseline-follow up design. In line with RDoC principles we use a strategy for recruiting all clients who "walk through the door" of a large community mental health clinic as well as the surrounding community. The clinic attends to a broad spectrum of anxiety and mood-related symptoms. Participants are unmedicated and studied at baseline using a standardized battery of functional brain imaging, structural brain imaging and behavioral probes that assay constructs of threat reactivity, threat regulation and cognitive control. The battery also includes self-report measures of anxiety and mood symptoms, and social and occupational functioning. After baseline assessments, therapists in the clinic apply treatment planning as usual. Follow-up assessments are undertaken at 3 months, to establish the reliability of brain-based subgroups over time and to assess whether these subgroups predict real-world functional capacity over time. First enrollment was August 2013, and is ongoing.

Discussion: This project is designed to advance knowledge toward a neural circuit taxonomy for mental disorder. Data will be shared via the RDoC database for dissemination to the scientific community. The clinical translational neuroscience goals of the project are to develop brain-behavior profile reports for each individual participant and to refine these reports with therapist feedback. Reporting of results is expected from December 2016 onward.

Trial registration: ClinicalTrials.gov Identifier: NCT02220309. Registered: August 13, 2014.

Keywords: Research Domain Criteria (RDoC), Brain circuits, Anxiety, Depression, Brain imaging, Emotion, Cognition

\section{Background}

Globally, one in 13 people suffer from clinical anxiety and about one in 21 suffer from clinical depression [1,2]. Anxiety and depression are the leading causes of disability and lost productivity worldwide [3]. Despite these alarming statistics, we still lack a valid classification system that links underlying neural mechanisms to individual symptoms, real-world functional consequences and the implications for treatment choices. In the current diagnostic system, the Diagnostic and Statistical Manual, fifth edition (DSM-5) [4] the broad categories of anxiety and mood disorders offer a reliable terminology for communicating between clinicians. The DSM-5 (and earlier editions) is not designed to reflect valid categories in terms of underlying neural function. At least $50 \%$ of people have concurrent diagnoses from more than one category of anxiety and mood disorder [5-7]. Due to the heterogeneity of these categories, it is also possible for two people to both be diagnosed with an anxiety disorder but share only one symptom, and the same is the case for depressive disorders. Individuals diagnosed with anxiety and depressive disorders, and their comorbidity, are also commonly treated with the same medications or behavioral therapies [8], reflecting our limited understanding of the distinct underlying mechanisms that could serve as targets for each intervention.

The National Institute of Mental Health has launched the Research Domain Criteria (RDoC) initiative, which is intended to advance the validity of mental disorder classification by incorporating neuroscience $[9,10]$. The ultimate goal of the RDOC initiative is to incorporate neuroscience in ways that will bridge the gap between research and clinical decision-making by helping to define cohesive subgroups relevant to treatment selection and to identifying targets for new interventions. The $\mathrm{RDoC}$ initiative is aligned with the vision for precision psychiatry [11] and offers an opportunity for an integrative understanding of mood and anxiety disorder [12].

Supported under the RDoC initiative, the present study is aimed at advancing a brain circuit-based taxonomy relevant to the spectrum of anxiety and depression. Brain imaging research in anxiety and depression has identified core nodes of large-scale circuits that are dysfunctional in these disorders. Here our primary focus is on two large-scale neural circuits found to be relevant to the phenotypes of anxiety and associated mood disorders [11]: the "negative affect" circuit and the "cognitive control" circuit (Fig. 1).

\section{"Negative affect" circuit}

The negative affect circuit is engaged by negatively valenced stimuli and comprises subcortical nodes in the amygdala, brainstem regions, hippocampus and insula and both dorsal and ventral prefrontal nodes - dorsal medial prefrontal cortex (dmPFC) and dorsal ACC connections as well as ventral mPFC (vMPFC) and ventral (subgenual and pregenual)rostral ACC connections [13, 14]. Dorsal/rostral nodes have been preferentially implicated in appraisal and expression of emotion and may be considered an "aversive amplification" sub-network [14] whereas the ventral nodes are implicated in automatic regulation of negative emotion $[13,15]$. These sub-networks may be engaged even in the absence of conscious sensory awareness, via direct brainstem inputs [16] (for meta-analysis [13]). In light of their commonly 


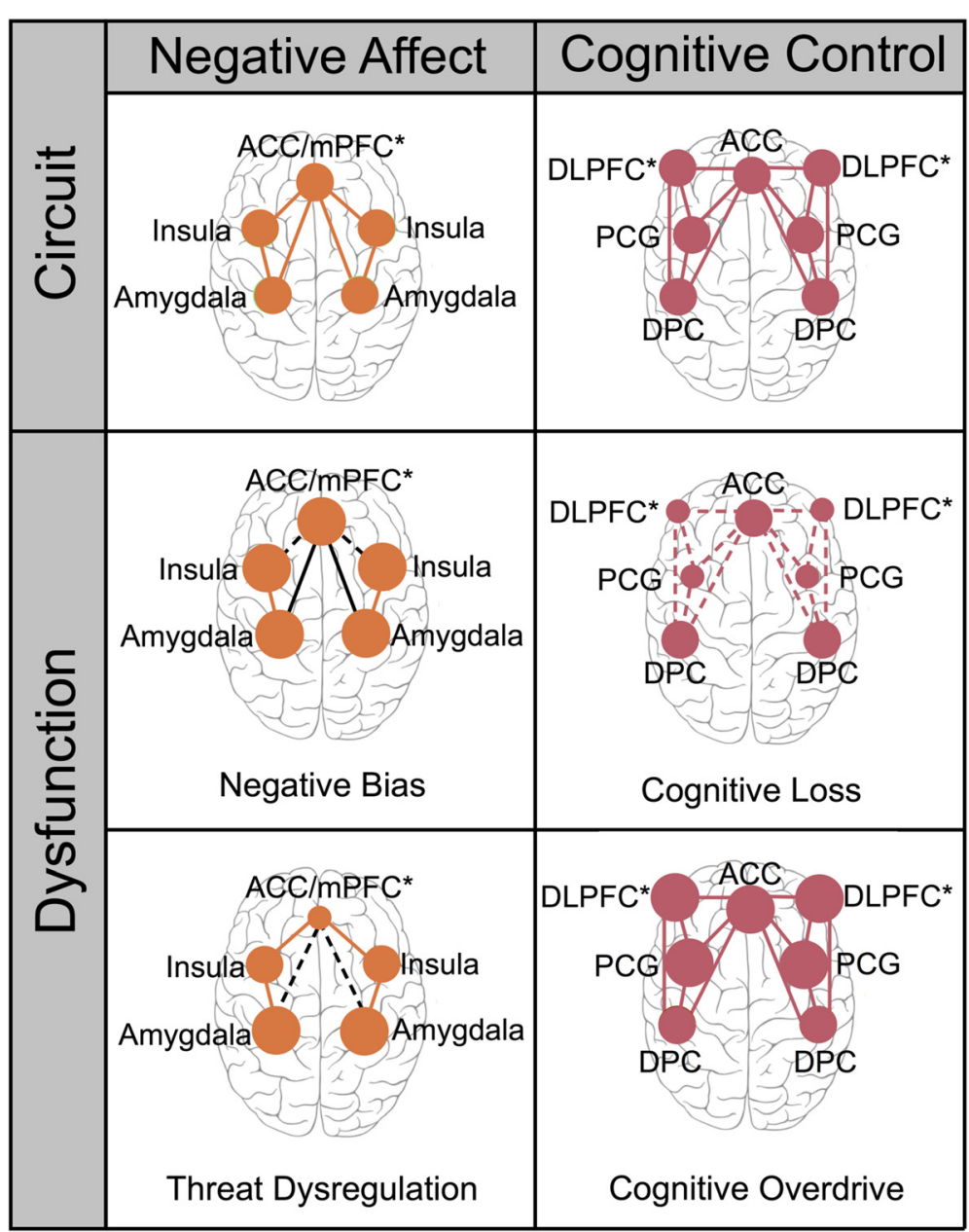

Fig. 1 Summary of negative affect and cognitive control circuits and the proposed extremes of dysfunction within these circuits

observed co-activation [13], the negative affect circuit could subserve the perception of negative emotion cues and the salience circuit, the arousal aspects of feeling these emotions.

\section{"Cognitive control" circuit}

The "cognitive control" circuit comprises the dorsolateral prefrontal cortex (DLPFC), anterior cingulate cortex (ACC), dorsal parietal cortex (DPC) and precentral gyrus [11]. Together these regions and their interconnectivity are implicated in the support of higher cognitive functions such as working memory and selective attention (for metaanalysis [17]), evidence from convergent neuroimaging methods [18]). Under task-specific demands the cognitive control circuit is implicated in cognitive flexibility [19].

In the current study we use these circuits as the independent (rather than dependent) variable to parse neural circuit-based types of dysfunction in a trans-diagnostic manner. We conceptualize types that represent extremes along dimensions of dysfunction within these circuits and their interactions.

\section{Proposed types of dsyfunction in the "Negative affect" circuit}

We hypothesize that negative affect circuit dsyfunctions will be expressed as hyper-reactivity in the bottom-up innervation of the amygdala and insula at one extreme and loss of top-down regulation of emotion-elicited reactivity at the other extreme (Fig. 1). These putative biotypes may contribute to subjectively experienced phenotypes of "negative bias" and "threat dysregulation" [11].

Amygdala hyper-reactivity elicited by non-conscious processing of masked threat stimuli has been reported in current depressive disorder (for review [20]), generalized anxiety disorder [21], generalized social phobia/anxiety disorder [21-23], specific phobia [24] and panic disorder $[21,24]$. Mood-congruent hyper-reactivity of the amygdala has also been observed in response to sad faces $[25,26]$. By contrast, the opposite finding of reduced amygdala activation for positive expressions has been observed in depressed people $[27,28]$. There are also findings of amygdala hypo- (rather than hyper-) reactivity for threat stimuli, which may characterize unmedicated participants 
who go on to respond to typical first-line antidepressants $[27,29]$.

Correspondingly, ACC hypo-activation during the processing of threat stimuli has been observed in generalized anxiety disorder $[15,30]$ and generalized social anxiety [30]. Reduced connectivity between the amygdala subgenual/ventral ACC has been observed during the processing of masked threat stimuli in unmedicated MDD [31], generalized social anxiety disorder [32] and generalized anxiety disorder [15].

\section{Proposed types of dsyfunction in the "Cognitive Control" circuit}

We hypothesize that cognitive control circuit dsyfunctions may also be expressed as hyper-reactivity at one extreme and hypo-activation reflecting loss of top-down control at the other extreme. These putative biotypes may contribute to subjectively experienced phenotypes of "cognitive overdrive" and "cognitive dyscontrol" [11] (Fig. 1).

Depressed people show hyper-activation of the DLPFC during working memory and executive function tasks, which may reflect an attempt at compensation to retain normal behavior (Fig. 1). DLPFC hyper-activation has been observed during tasks with an increasing cognitive demand, but in the absence of performance deficits, in, medicated MDD [33] and unmedicated MDD [34]. Hyper-activation of the ACC has also been observed in MDD when participants are performing similarly to controls (rostral ACC [33]) and this effect persists after remission.

Dysfunction of the cognitive control circuit may be elicited by tasks requiring effortful selective processing of relevant while inhibiting irrelevant stimuli, and suggests a "cognitive loss" type (Fig. 1). Hypo-activation of the DLPFC and dorsal anterior cingulate cortex (dACC) has been observed across diagnoses, including depression [35-37] and social anxiety [38]. Correspondingly, depression has been associated with a loss of functional DLPFC-dACC connectivity when cognitive control is required [36]. Hypo-activity in these nodes of the cognitive control circuit has been found to persist after recovery in adult and later-life depression [39] suggesting that a "cognitive loss" type may have a trait-like status.

\section{Linking brain circuit dysfunction to symptom profiles}

It is not yet known how dysfunctions involving the negative affect and cognitive control circuits relate to specific features of anxiety and mood symptoms. Here we assess multiple features of anxiety, depression and general distress, encompassing constructs of "anxious arousal", "apprehensive expectations" (and rumination) and anhedonia. Paralleling the findings for brain imaging of neural circuits, a wide range of disorders share common symptoms. For example, multiple anxiety disorders (e.g., specific phobia, panic and social anxiety) share symptoms of anxious arousal (implicating dysfunctions in bottom-up threat reactivity), while other general and trauma-related anxiety disorders (GAD and PTSD) are accompanied by more cognitive experiences of anxiety, such as apprehensive expectations (implicating lack of emotion regulation) [40, 41]. Data-driven techniques such as factor analysis may be useful in identifying specific dimensions of symptoms that cut across traditional diagnoses [42].

The RDoC Anxiety and Depression ("RAD") study is supported by the NIMH RDoC initiative, and designed to make progress toward a cohesive brain-based taxonomy relevant to the spectrum of anxiety and associated mood disorders and associated impairments in behavior and real-world function.

\section{Research objectives \\ Primary objectives}

1. To use brain imaging to identify cohesive transdiagnostic dimensions of dysfunction in the "negative affect" circuit for threat reactivity and regulation, and associated dysfunctions in the "cognitive control" circuit. We will also assess interactions between these brain circuit dysfunctions.

2. To assess whether brain circuit-defined dysfunctions relate to the severity of specific anxiety and mood symptoms, such as anxious arousal and apprehensive expectations.

3. To assess whether these brain circuit-defined dysfunctions also relate in a cohesive way to behavioral performance and to real world functions related to burden of illness, specifically social functioning, quality of life and work productivity.

\section{Secondary objective}

To assess if the brain circuit-defined dimensions predict change in symptoms and real world functions over time.

\section{Ethical approval}

The Institutional Review Boards of Stanford University and Palo Alto University have approved this protocol. Informed written consent and permission to publish any direct quotes from interviews will be obtained from each participant. Consent will also be obtained to video-record feedback sessions to be used for training purposes.

\section{Methods and design}

We use a baseline-follow up design. Intensive assessments for all measures of interest are undertaken at the baseline session and after 12 weeks participants are re-assessed at follow-up on the symptom and functional outcome measures (Table 1). We control for medication-free status at 
Table 1 A summary of the test-retest design and type of data collected at each assessment time point

\begin{tabular}{lll}
\hline Screening & Baseline & Follow-up \\
\hline $\begin{array}{l}\text { Self-reported } \\
\text { symptoms }\end{array}$ & $\begin{array}{l}\text { Structured Clinical } \\
\text { interview }\end{array}$ & \\
$\begin{array}{l}\text { Contraindications } \\
\text { for medication }\end{array}$ & $\begin{array}{l}\text { Brain imaging of } \\
\text { circuits }\end{array}$ & \\
& $\begin{array}{l}\text { Behavioral } \\
\text { performance }\end{array}$ & \\
Contraindications & $\begin{array}{l}\text { Self-reported } \\
\text { for brain imaging }\end{array}$ & $\begin{array}{l}\text { Self-reported } \\
\text { symptoms }\end{array}$ \\
$\begin{array}{ll}\text { Other contraindications } \\
\text { for study participation }\end{array}$ & $\begin{array}{l}\text { Self-reported and } \\
\text { observer-rated coping, } \\
\text { social and occupational } \\
\text { function }\end{array}$ & $\begin{array}{l}\text { Self-reported and } \\
\text { social and occupational } \\
\text { function }\end{array}$ \\
\hline
\end{tabular}

the baseline session and record in an observational (rather than experimental) manner any interventions that participants were exposed to in the 12-week period between baseline and follow up. Because this was not an intervention study, but rather an investigation of the natural stability of brain-behavior-symptom relationships over time we do not control treatment type or intensity. Our clinical center partners use a structured treatment planning approach based on integrative psychotherapy principles with pharmacological augmentation and all participants recruited from the center are currently receiving individual therapy.

Participants are free of antidepressant medications and other medications that could impact the brain imaging assessments.

Consistent with the goal of $\mathrm{RDoC}$ and characterizing brain imaging-derived (rather than diagnostic) constructs, screening and exclusion criteria are kept to a minimum. We do not exclude for cognitive indicators of potential mild cognitive impairment (MCI) given that such cognitive deficits in many patients are associated with their anxiety and mood symptoms but are not indicative of neurodegeneration (for review; [43]). These indicators are recorded for inclusion as covariates in analyses.

For participants who meet inclusion criteria and are not screened out by exclusion criteria we schedule a testing site visit. At the testing site visit brain imaging, behavioral and self-report/observer-rated assessments are completed (Table 1).

\section{Recruitment}

We aim to enroll 160 participants who comprise a clinicalcommunity sample. Enrollment commenced in August 2013, and we anticipate enrollment for baseline assessments will be completed in 2017.

We are enrolling patients from the Gronowski Center (GC), a community mental health training clinic, and individuals from the immediate surrounding community.
The target of 160 ensures sufficient statistical power and coverage of the spectrum of anxiety and associated mood symptoms. Our power calculation is designed to test for an anticipated regression model with a medium effect size; approximately .5 (or $\mathrm{f}^{2}=.0625$ ). With an alpha level of .05 , a power level of .80, two primary predictors (our R0Is) and four additional predictors (behavioral and questionnaire measures) we require at least 158 participants. We have anticipated screening at least 3 times the target number in order to achieve a total enrolled sample of 160.

We are not using psychiatric diagnosis as inclusion criteria. We include participants spanning the dimension from healthy through sufficiently severe to meet diagnostic criteria.

To demonstrate the spread of coverage from our recruitment strategy, we record diagnosis (or absence of diagnosis), but do not use this information for primary analyses. Based on prevalence data from the existing GC records, we have anticipated the following spectrum of diagnoses: social anxiety disorder (6\%), generalized anxiety disorder (13\%), panic disorder (3\%), agoraphobia or specific phobia (1\%), post-traumatic stress disorder (8\%), obsessive compulsive disorder (4\%), major depressive disorder (26\%), persistent depressive disorder (dysthymia) (12\%), bipolar disorder 1 (5\%), other specified or unspecified depression or anxiety (5\%).

\section{Inclusion and exclusion criteria}

Inclusion criteria are: i) age (18+ years) to focus on the adult brain, ii) fluent and literate in English in order to understand task instructions, and iii) currently reporting distress from anxiety and related mood symptoms. Exclusion criteria are: i) current or lifetime experience of frank psychosis and/or mania, because the circuit dysfunctions associated with such phenomenology might obscure interpretation of anxiety and mood-related circuit dysfunctions, ii) presence of suicidal intent representing imminent risk as indicated during screening and on-site assessments, iii) medical condition or neurological disorder that could impact brain imaging data and render images difficult to interpret, iv) history of physical brain injury or blow to the head resulting in loss of consciousness greater than five minutes and which in the judgment of investigators could interfere with interpretation of brain imaging assessments, and v) severe impediment to vision, hearing and/or hand movement, likely to interfere with the ability to complete the assessments, or follow the instructions.

We record information about comorbid conditions that are not part of the exclusion criteria, including substance use and other general medical conditions. In regard to substance use disorders, we accept participants who are using alcohol and substances as a comorbid behavior 
given the emphasis on recruiting a representative sample. A NIH Certificate of Confidentiality that has been granted to the principal investigator protects participants.

\section{Screening}

Participants are screened over the phone during a call that lasts about $15 \mathrm{~min}$. Oral consent is taken, and study details are explained. Questions about participant demographics and inclusion and exclusion criteria are asked. Inclusion criteria questions include being at least 18 years of age, having recent symptoms of anxiety or depression, and speaking English. Exclusion criteria questions include recently taking psychiatric medications, traumatic brain injuries, MRI contraindications (e.g., implanted devices, pregnancy, claustrophobia, ferromagnetic material in the body), and psychosis. At the end of the screening interview, a determination of the participant's eligibility is made with the approval of the principal investigator.

\section{Baseline visit}

At the baseline visit we undertake a traditional clinical interview, using the Mini-International Neuropsychiatric Interview (MINI-Plus) [44]. We also assess demographics (including age, sex, education and handedness using the Edinburgh Handedness Inventory), and prior and current medical status. Detailed assessments targeting our primary aims are also undertaken at the unmedicated baseline visit, and Table 2 provides a tabular summary of these measures.

\section{Clinical interview, medical history and demographics} At baseline the MINI-Plus [44] is used to assess DSM-IV criteria for anxiety and mood disorder and to confirm exclusions due to psychosis and/or clinically significant mania. To ensure currency we also apply DSM-5 criteria to the information gathered at interview. Research personnel also gather comprehensive information on past and current medical history and additional sociodemographic data.

\section{Brain imaging}

Participants undergo a brain scan protocol that will include a battery of previously established standardized paradigms [45].

\section{Paradigms}

i) Viewing of threat faces We selected the masked viewing of threat faces paradigm because it reliably engages negative affect circuitry, is grounded in proposed mechanisms of anxiety, and has been well established by the investigators. In the masked condition this paradigm probes automatic bottom-up activation of the negative affect circuit $[16,46,47]$. Stimuli are from a standardized series of facial expressions of threat-related emotions (fear, anger), loss related emotions (sadness) and reward-related emotions (happiness), along with neutral, modified such that the eyes are presented in the central position of the image [48]. Stimuli are presented for $16.7 \mathrm{~ms}$, determined to be below the sensory threshold for conscious identification of emotion, followed immediately

Table 2 A summary of the measures used in the trial to assess circuit function, behavior and self-report and observer rated symptoms and real-world function

\begin{tabular}{|c|c|c|c|}
\hline fMRI Paradigms & Behavioral Tasks & Self and Observer Report & \\
\hline Negative Affect Circuit & & Clinical Measures & Real World Function \\
\hline Masked Facial Expressions of Threat & Threat Identification and Bias & Symptoms of anxiety and negative mood: & Coping: \\
\hline \multirow[t]{3}{*}{ Threat Conflict Regulation } & \multirow[t]{3}{*}{ Threat Conflict Adaptation } & \multirow[t]{3}{*}{ BAI, PSWQ, BDI, QIDS-SR, DASS, MASQ } & COPE \\
\hline & & & Emotion Regulation: \\
\hline & & & $\mathrm{ERQ}$ \\
\hline Cognitive Control Circuit & & $\begin{array}{l}\text { Symptoms of associated traumatic stress, } \\
\text { impulsivity, and substance use: }\end{array}$ & Social and Occupational Function: \\
\hline \multirow[t]{3}{*}{ Go-NoGo } & \multirow[t]{3}{*}{ Go-NoGo } & \multirow{3}{*}{$\begin{array}{l}\text { PCL-C, BIS, FTND, DSM } 5 \text { AUD, DSM } 5 \text { SUD, } \\
\text { AUDIT, CUDIT, NM-ASSIST }\end{array}$} & SOFAS \\
\hline & & & Quality of Life: \\
\hline & & & WHOQOL, SWLS \\
\hline \multirow[t]{2}{*}{ N-Back Continuous Performance } & Digit Span & Trauma-related risk: & Work productivity: \\
\hline & Verbal Learning and Memory & ELSQ & $\mathrm{HPQ}$ \\
\hline
\end{tabular}

Abbreviations: BAI Beck Anxiety Inventory, PSWQ Penn State Worry Questionnaire, BDI Beck Depression Inventory, QIDS-SR Quick Inventory of Depressive Symptomatology-Self Report, DASS depression anxiety stress scales, MASQ Mood and Anxiety Symptom Questionnaire, PCL-C Posttraumatic Stress Disorder CheckList-Civilian Version, BIS Barratt Impulsivity Scale, FTND Fagerstrom Test for Nicotine Dependence, DSM 5 AUD DSM 5 Alcohol Use Disorder, AUDIT alcohol use disorders identification test, DSM 5 SUD DSM 5 substance use disorder, NM-ASSIST NIDA-Modified Alcohol, Smoking and Substance Involvement Screening Test, CUDIT Cannabis Use Disorder Identification Test, ELSQ Early Life Stress Questionnaire, COPE Brief COPE, ERQ Emotion Regulation Questionnaire, SOFAS Social and Occupational Functional Assessment Scale, WHOQOL World Health Organization Quality of Life Scale, SWLS satisfaction with life scale, HPQ World Health Organization Health and Work Performance Questionnaire 
by a neutral face perceptual mask for $150 \mathrm{~ms}$ and an interstimulus interval of $1233.3 \mathrm{~ms}$. Mask stimuli are offset by $1^{\circ}$ in the direction of each diagonal, randomly, in order to control for the potential effects of priming due to the perceptual difference between emotion-neutral and neutral-neutral target-mask pairs. Using a blocked design, stimuli will be grouped with eight faces expressing the same emotion per block and repeated 5 times in a pseudorandom order [45]. Using behavioral psychophysical testing, we have shown that when faces in this paradigm are presented at or below $20 \mathrm{~ms}$, they meet signal detection criteria for being at the subliminal threshold for detection, such that individual participants cannot detect the presence of the face nor discriminate the facial expression [48].

To provide a positive control for the masked condition we also present the same 240 standardized facial expressions described above in an explicit conscious perception condition. In this condition stimuli will be presented for $500 \mathrm{~ms}$, with an interstimulus interval of $750 \mathrm{~ms}$, also in a blocked design. The stimulus duration of $500 \mathrm{~ms}$ was based on evidence that this is sufficient time to allow for conscious elaborative processing of the emotion stimulus. Conscious discrimination of emotion is consistently above chance (and close to $100 \%$ ) at durations $\geq 330 \mathrm{msec}$ [48] and facial expressions of emotion consistently elicit a contagious effect of experiencing the emotion signaled by the stimulus at durations of $500 \mathrm{~ms}$ [49]. Stimulus onset asynchronies are standardized at $1250 \mathrm{~ms}$ across both masked and explicit perception paradigms. No specific behavioral responses were required during scanning because of the inclusion of subliminal presentations and our aim to isolate activation elicited by emotion stimuli independent of behavioral task demands. A large meta- analysis of 385 studies has shown passive processing is associated with a higher probability of activation than an active task [50]. We created a context for participants to continuously view the faces by instructing them that they would be asked post-scan questions about these faces. We will control for active attention to the face stimuli by monitoring alertness with an eye tracking system.

ii) Threat conflict regulation paradigm We selected the threat conflict paradigm because it also reliably engages negative affect circuitry, in this for top-down regulation of emotional reactivity, and is also well established by the investigators $[15,51]$.

In the threat regulation task participants are presented with a total of 148 happy or fearful facial expressions [52], while ignoring an overlying word labeling the expression ("FEAR" or "HAPPY"). The word either matches the facial expression (congruent) or conflicts with it (incongruent). Each stimulus will be presented for $1000 \mathrm{~ms}$ with a variable interstimulus interval (mean: 4000; range: 3000-
$5000 \mathrm{~ms})$. Stimuli will be presented in a psudorandom order [51].

iii) Go-NoGo paradigm We use the Go-NoGo paradigm that has been established as a robust probe of the cognitive control circuit $[45,53,54]$. The Go-NoGo paradigm is used to assess impulsivity (automatically-generated 'Go' responses) versus inhibition ('NoGo' responses). In the 'Go' trials, participants are required to "press" on GREEN stimuli (the word "press"), while in the 'NoGo' trials; participants withhold presses on RED stimuli. Stimuli are presented for $500 \mathrm{~ms}$ each with an interstimulus interval of $750 \mathrm{~ms}$. Participants are asked to respond via button press as quickly as possible to the Go stimuli and inhibit their response for the NoGo stimuli. Reaction times and number of errors on task are used to evaluate task performance. The design of this paradigm allows for event-related analysis. The probability of NoGo stimuli is .33. There are a total of 180 Go and 60 NoGo stimuli presented in a pseudorandom order with a constraint to ensure that NoGo stimuli are not repeated more than 3 times in a row.

iii) N-back Continuous Performance Test As a complement to the Go-NoGo paradigm used to assay cognitive control functions we also use an n-back Continuous Performance Test (CPT) to assess sustained attention and selective working memory updating [45]. In the "sustained attention" target trials, participants press a button when the same letter appears twice in a row (a '1-back' design). In the "working memory updating" trials participants are required to continually update the contents of working memory in case of a target. In total there are 120 letters (B, $\mathrm{C}, \mathrm{D}$ or G) presented sequentially, including 20 sustained attention targets in yellow, 60 working memory updating stimuli in yellow and 20 addition "to be ignored" letter in white which were intended to provide a perceptual baseline condition. Stimuli are presented for $200 \mathrm{~ms}$ each with an inter-stimulus interval of $2300 \mathrm{~ms}$. Presentation was pseudorandom, ensuring there were no consecutive target tones. One fMRI volume per stimulus was acquired.

\section{Acquisition and quantification}

i) Functional scans For each paradigm, blood oxygenation level-dependent contrast functional images are acquired with echo-planar T2*-weighted imaging using 3.0 Tesla GE Signa HDx scanner (GE Healthcare, Milwaukee, Wisconsin) with a 32-channel head coil. Each whole brain volume will consist of 45 interleaved $3 \mathrm{~mm}$ thick axial/oblique slices ( $74 \times 74$ matrix; TR, $2000 \mathrm{~ms}$; TE, $27.5 \mathrm{~ms}$; size, $3 \times 3 \times 3 \mathrm{~mm}$; FOV, $222 \mathrm{~mm}$; flip angle, $\left.77^{\circ}\right) .154$ volumes will be acquired over $5.03 \mathrm{~min}$ and $8 \mathrm{~s}$ for all paradigms but the emotion regulation task. For the 
emotion regulation task a total of 397 volumes will be collected over $13 \mathrm{~min} 14 \mathrm{~s}$. Three dummy scans are acquired at the start of each acquisition.

Preprocessing and data analysis is performed using Statistical Parametric Mapping software implemented in Matlab (SPM8; Wellcome Department of Cognitive Neurology) in a manner similar to that of our prior publications [27, 45]. Specifically, Motion correction is performed by realigning and unwarping the fMRI images to the first image of each task run. Following realignment and unwarping, quality control diagnostics are completed on the time series data for each run. Images are normalized to the stereotactic space of the Montreal Neurological Institute (MNI) template [55], T1- weighted data are normalized to standard space using the FMRIB nonlinear registration tool and the fMRI EPI data are coregistered to the T1 data using FMRIB linear registration tool. Normalization warps from these two steps are stored for use in functional to standard space transformations. Global signal is estimated using a eroded mask within the ventricles and white matter and is removed from the motioncorrected fMRI time series. fMRI data are smoothed using an $8 \mathrm{~mm}$ Gaussian kernel and high-pass filtered using a cutoff period of $128 \mathrm{~s}$.

To define circuit-based constructs we focus first on specific nodes in the circuits of interest using a region of interest (ROI) approach, established previously [56]. With the RO1 approach we identify BOLD-dependent signal change in the defining nodes of the negative affect circuit, including amygdala, insula, ACC/mPFC (ventral and dorsal). Beta values for each ROI are extracted for each subject for regression analyses. We will also use functional connectivity analyses to quantify the functional relationships between regions. Further, exploratory whole brain, voxel-wise analyses are conducted using a significance threshold of $\mathrm{p}<0.05$ corrected for multiple comparisons. Regions of activation are defined according to the Talairach Atlas. In parallel we study additional regions as part of the exploratory goals of the study.

ii) Additional exploratory functional analyses Since we recognize that the amygdala and ACC are part of more extensive circuits, in the exploratory phase we will also assess activation in other regions, informed by the extant literature and any new developments in the field.

iii) Structural scans A high-resolution T1-weighted structural scan is acquired using a 3D spoiled gradient echo (SPGR) sequence at the end of the imaging session for use in normalization of the fMRI data into standard space. A diffusion scan is also acquired in order to quantify white matter integrity.

\section{Behavior}

\section{Computerized tests of behavioral performance}

Threat identification and bias: The same faces as shown during fMRI are presented on a computer screen (96 stimuli, 8 different individuals). Identification is recorded by the verbal labeling of the expressions and the reaction time to do so. Implicit priming of reaction time to "old/ new" memory recognition of faces, primed by prior exposure to facial expressions of threat versus neutral, to elicit biases to threat using an established protocol [57]. The bias to fear is the reaction time difference (in milliseconds) for priming due to threat minus neutral.

Threat conflict adaption: The Emotional Conflict task generates reliable reaction time interference $[15,51,58]$. We will quantify reaction time for successive trials, indexing adaptive regulation to fear-related conflict.

Go-NoGo: To assess response inhibition we use a previously established task in which participants respond quickly to green stimuli and withhold responses to red stimuli [59].

We will also assess cognitive control using additional behavioral tests of executive function, memory and inhibition, including the following:

Digit span. To assess working memory participants are asked to hold online a span of 2 to 9 digits and then repeat these digits in order.

Verbal learning and memory. To test immediate and delayed recall of verbal information (12-word lists), equivalent to the constructs assessed by the California Verbal Learning and Memory test.

Verbal Interference test: Using previously established tests assessing constructs equivalent to those assessed by the Stroop color/word test [59].

\section{Acquisition and quantification}

Each of these behavioral tests runs on a desktop computer, and does not rely on keyboard skills. The software used to run the tasks includes standardized task instructions. Psychometric properties have been established for each of these tests, including norms, construct validation, validation against traditional neuropsychological tests tapping equivalent functions, test-retest reliability, and consistency across cultures [59-64]. The tests have been used effectively in patient groups in previous research by the investigators [65-68].

For each test we record accuracy and reaction time via the testing software. These data are logged in a file on the desktop computer under the identification code for each participant.

\section{Self-report and observer-rated measures Symptoms of anxiety and negative mood}

i) Beck Anxiety Inventory (BAI) A 21-item self-report inventory for measuring the severity of common symptoms 
of anxiety that the participant has had during the past week, such as numbness and tingling, sweating not due to heat, and fear of the worst happening [69].

ii) Penn State Worry Questionnaire (PSWQ) A 16-item questionnaire that assesses items such as "my worries overwhelm me" and is rated on a likert scale, with scores ranging from 1 to 80 [70].

iii) Beck Depression Inventory (BDI) A 21-item, selfreport rating inventory that measures symptoms of depression such as hopelessness and irritability, cognitions such as guilt or feelings of being punished, as well as physical symptoms such as fatigue, weight loss, and lack of interest in sex [71].

iv) Quick Inventory of Depressive Symptomatology, Self-Report (QIDS-SR) A 16-item self-report assessment of the nine DSM-IV symptom criteria for major depressive disorder. Individuals rate items as present, mild, moderate severe, and scores range from 0 to 27 [72].

v) Depression, Anxiety and Stress Scale (DASS) The DASS is a 42-item self-report scale that assesses symptoms of depression/anhedonia, anxious arousal and generalized anxiety (stress) that are not tied to a particular diagnosis. The DASS has been normed for use in healthy and patient groups, and validated against other measures of anxiety such as the BAI described above [73].

iv) Mood and Anxiety Symptom Questionnaire (MASQ) A 90-item questionnaire based on the tripartite model of affective disorder which encompasses constructs of anhedonia, anxious arousal and generalized distress, equivalent to the DASS, and which has also been used in healthy and patient groups [74].

\section{Symptoms of associated traumatic stress, impulsivity and} substance use symptoms

i) PTSD CheckList - Civilian Version (PCL-C) A selfreport rating scale for assessing the 17 DSM-IV symptoms of PTSD. This version is a general civilian version that is not linked to a specific event. A total score is computed by adding the 17 items, so that possible scores range from 17 to 85 [75].

ii) Barratt Impulsiveness Scale (BIS) A questionnaire designed to assess the personality/behavioural construct of impulsiveness [76] that may reflect subclinical mania and related experiences. iii) Fagerström Nicotine Dependence Scale (FNDS) A short 6-item instrument used for assessing the intensity of physical nicotine addiction [77].

iv) DSM-5 alcohol use disorder A 12-item questionnaire assessing alcohol use based on the DSM-5 criteria [4].

v) Cannabis Use Disorder Identification Test - Revised (CUDIT-R) A cannabis misuse screening tool containing 8 items, two from each of the domains of consumption, cannabis problems (abuse), dependence, and psychological features [78].

vi) DSM-5 substance abuse disorder An 11-item questionnaire assessing substance use disorders based on the DSM-5 [4].

vii) NIDA-Modified Alcohol, Smoking, and Substance Involvement Screening Test (NM-ASSIST) A 15-item measure adapted from the World Health Organization (WHO) Alcohol, Smoking and Substance Involvement Screening Test, used to assess prescription medicine and illicit substance use in adults age 18 and older [79].

\section{Trauma-related risk factors}

Early Life Stress Questionnaire (ELSQ): A 19-item questionnaire used to retrospectively assess exposure to early life stress, by ascertaining whether the participant had experienced physical, emotional, or sexual abuse as well as other traumatic experiences such as sustained bullying, poverty, divorce, illness, or domestic violence. If a stressor was present, the participant identified age of onset $(0-12$ years of age) [80].

\section{Real world function and coping}

i) Brief COPE A multidimensional coping inventory to assess the different ways in which people respond to stress [81].

ii) Emotion Regulation Questionnaire (ERQ) A 10-item questionnaire designed to assess individual differences in the habitual use of two emotion regulation strategies: cognitive reappraisal and expressive suppression [82].

iii) Social Functioning and Adjustment Scale (SOFAS) The SOFAS is a derivative of the Global Adjustment Scale and reflects the individual's level of social and occupational functioning, rated on a scale from between 0 and 100 [83].

iv) World Health Organization Quality of Life (WHOQOL) scale This is a 30-item scale that assesses the psychological, general health, physical and environmental 
aspects of quality of self-reported quality of life, each assessed out of 100 [84].

v) Satisfaction With Life Scale (SWLS) A short, 5-item instrument designed to measure global cognitive judgments of satisfaction with one's life [85].

vi) Health Productivity Questionnaire (HPQ) This is a 14-item scale that assesses productivity at work, and relative and absolute levels of absenteeism from work as well as presenteeism (being at work, but unproductive due to the effects of anxiety and depressive symptoms [86].

\section{Acquisition and quantification}

Each self-report measure is presented in a computerized format. Individual item scores are recorded and then summed automatically according to symptom cluster and scale definitions. The only exception to automated scoring is the SOFAS, which is rated by study coordinators.

\section{Follow-up outcome measures}

At 12-week follow-up, we assess change in/stability of symptoms using the PSWQ, DASS and MASQ, HPQ and WHOQoL.

\section{Translational outcomes: toward a precision mental health protocol}

Participants are offered the opportunity to partake in a brief feedback session (i.e., $30 \mathrm{~min}$ ) with the study principal investigator, key clinical personnel and their current therapist. The feedback is based on self-report measures such as mood and anxiety symptoms, social and occupational functioning, and behavioral tests of emotion and cognition. A brief clinical summary of the participant's history is provided to the investigator as contextual information. The investigator discusses the participant's profile on the measures, describes relevant test performance, and gives recommendations for how this information could be applied to treatment. Anxiety and depression are examined from an $\mathrm{RDoC}$ perspective incorporating such dimensions as threat reactivity, threat regulation and cognitive performance; the participant is offered an overview of their experience based on a neural circuitry perspective. The participant and their therapist are given the opportunity to ask questions regarding current symptomatology as related to the neuroimaging and behavioral data. At the conclusion of the feedback session, the investigator exits while the participant and therapist consider the information received. The ultimate goal is to collaborate on incorporating the feedback into the current treatment plan. Treatment outcomes such as symptom reduction and therapy retention will be examined for participants who receive the feedback.

\section{Data management}

All data are de-identified using participant numbers and no session number is identified. Follow-up data is distinguished from baseline visit data with timestamps and distinct variable names.

Source documents will be archived for 7 years beyond study completion, or in accordance with IRB regulations, whichever is longest.

Neuroimaging data are managed using the quality control and data management infrastructure at the Stanford Center for Neurobiological Imaging (CNI). At CNI, the Neurobiological Image Management System (NIMS) has been in use for the past four years. The current version of the software supports standardized format (DICOM) files. NIMS was designed to address key issues in order support the principles of reproducible research by i) enabling data sharing in way that is consistent with current norms in the field of neuroscience and ii) combining imaging data and metadata about subjects in a searchable database system.

For the computerized behavioral tests, the computer registers each response and writes these with time stamps to a $\log$ file. For the questionnaires, each selfreported response entered by participants is logged. These data are stored in a PHI-protected database and then integrated with the neuroimaging data.

In accord with the requirements of the project funding award, the data will be shared via the NIMH RDoC database, RDoC-db.

\section{Statistical analysis}

The statistical analysis plan is designed to test specific working hypotheses relevant to each of the primary aims of the study:

1. We will use both a priori approaches to group participants into "high" versus "low" subgroups on each imaging circuit measure, and their combination. We will also use data-derived techniques such as cluster and factor analysis to complement these a priori approaches.

We will use General Linear Models (GLMs) to compare "high" versus "low" brain circuit-derived subgroups and data-derived clusters on symptom profiles. In correlational models we will assess the relationships between brain imaging dimensions and specific symptom profiles.

2. We will use GLMs to compare brain imaging-derived subgroups/clusters on behavioral performance. We will also test associations between behavior, imaging and symptoms using correlational and mediator/moderator models.

3. We will use GLMs to compare brain imaging-derived subgroups/clusters on real-world function. We will 
also test associations between function, imaging, behavior and symptoms using correlational and mediator/moderator models.

The statistical analysis plan also addresses the secondary aims of the study:

4. Repeated-measures GLMs, reliability and regression models will be used to assess change (or stability) in symptoms and function at follow-up and to test if imaging at baseline predicts profiles of symptoms and function after 12 weeks.

We will use multiple methods to integrate the data under each aim.

As noted above we will use mediator and moderator approaches to model the relationship between imaging, symptoms, behavior and/or function at baseline and over time.

Second, we will employ multiple methods for implementing data reduction and pattern discovery, depending on the dimensionality and distributions of data domains. For example, we will employ model-based sparse factor analysis [87], a method that has been developed and successfully employed on functional neuroimaging data. We will do so hierarchically, to find simple structure within each domain separately, and then across domains, using Bayesian Belief Nets [88] to obtain directed relationships. We will also examine associations between domains using modern variable selection techniques such as the Elastic Net [89], a combined regression and variable selection methodology that is capable of handling large numbers of correlated explanatory variables. Finally, we will use techniques such as canonical correlation analysis to determine linear combinations of variables in given domains (e.g., neuroimaging) that maximally relate to variables in other domains (e.g., behavioral or symptoms).

\section{Power calculation}

Power is estimated for GLMs assuming a small effect size of Cohen's $f^{2}=0.10$. With 160 subjects (after dropout and exclusions) and with moderately correlated predictors (average $\mathrm{r}=.5$ ) after multiple testing adjustments, we have well over $80 \%$ power to detect effect with family-wise corrected alpha level of $\mathrm{p}<.05$ [90].

\section{Discussion}

The project aligns tightly with the goals and units of measurement defined by RDoC [10], to advance a neurobiologically informed understanding of mental disorders. Currently our understanding of the brain basis of anxiety and mood disorders is limited, especially at the individual patient level. To our knowledge, no previous study has characterized types of brain circuit dysfunctions across disorders and then evaluated how these dysfunctions relate to specific symptom profiles, behaviors and real-world functioning. Defining these types of dysfunction and relationships in a cohesive way will provide a foundation for ultimately tailoring interventions to individual needs.

We suggest that, to move forward in understanding the mechanisms of anxiety and depression and potentially improve quality of life, a shift is required to understanding the brain circuit domains linked to observed symptoms, behavior and function. Given that anxiety and depression are so prevalent $[1,2]$ generate significant disability [3] and we do not yet have biomarkers to help guide treatment decisions in clinical settings this study addresses an important public health need to understand these disorders from a neurobiological rather than diagnostic point of view - in order to better explain the mechanisms by which dysfunction occurs and to better treat these disorders.

Strengths and innovation of this project include the use of mechanistically delineated paradigms for probing brain circuits and behavior such that brain-based constructs can be interpreted in the context of their behavioral expression as well as symptoms, the grounding in a conceptual model that goes beyond simply emotion processing to cognition and real world function, the use of well-established standardized assessments, and the clinical partnership that ensures recruitment of a sample representative of the community of people with anxiety and mood disorders.

\section{Limitations and challenges}

We are conscious that recruitment of participants from community settings is a complex undertaking, which requires us to consider the clinical priorities of therapists and center supervisors. There is also a need to ensure a smooth flow for testing visits and to minimize participant burden. We have optimized the flow by using standardized assessments that minimize assessment time and by establishing a participant-friendly environment for the assessments. We also ensure that one research coordinator maintains contact with each participant to individualize the experience and to maximize retention in the study. Data collection will generate a large volume of data, and we have sought to address data management issues by establishing an efficient server infrastructure.

Despite these challenges, the project is expected to have an immediate impact on delineating the brain circuits and behavior that define cohesive constructs within the spectrum of anxiety and mood disorders, resulting in a new approach to diagnoses of anxiety and mood and provide a foundation for future tailoring of treatments to individual needs. 


\section{Endnotes}

${ }^{1}$ The NIMH award title for this project is "Neural dimensions of threat reactivity and regulation for understanding anxiety"

\section{Competing interests}

In the past three years AE has received research project fees from Brain Resource and AE has received consultant fees from Otsuka. LMW has received consultant fees from Humana and has served on the scientific advisory board of Psyberguide. AS has served as a consultant to BrainCells, CeNeRx, CNS Response, Depomed, Eli Lilly, Forest Labs, Genetech, Gilead, GSK, Jazz, Lundbeck, Merck, Neuronetics, Novadel, Novartis, Pathway Diagnostics, Pfizer, PharmaNeuroBoost, Quintiles, Sanofi-Aventis, Sunovion, Synosia, Takeda, Xytis and Wyeth. AS has equity in Amnestix, BrainCells, CeNeRx, Corcept (co-founder), Delpor, Forest, Merck, Neurocrine, Novadel, Pfizer, PharaNeuroBoost, Somaxon, Synosis, and Titan. He is a named inventor on pharmacogenetic use patents on glucocorticoid antagonists and on prediction of antidepressant response and has received speaking fees from Merck, GlaxoSmithKline and Roche. In the last three years, TS has received funding from National Institute of Mental Health, Sunovion Pharmaceuticals, Elan Pharma and VA Cooperative Studies. She has also served as a consultant/ speaker to Lundbeck, Sunovion, Aztra Zeneca, Merck, Medscape Education, Omnia-Prova Education Collaborative, and Global Medical Education. Additionally, she has received royalties from Jones and Bartlett and UpToDate. $\mathrm{NC}, \mathrm{KG}, \mathrm{NH}, \mathrm{AG}, \mathrm{ROH}$ and JY report no competing interests.

\section{Authors' contributions}

LMW is the principal investigator, who conceived the study, obtained funding, designed and coordinated the study and drafted and revised the manuscript. AE, ROH, AFS, TS and JY are co-investigators who contributed to the design and conduct of the study and revised the manuscript. $\mathrm{NH}$ is a key collaborator who contributed to the conduct of the study, and participant recruitment in relation to the partnership with the Gronowski Center, and revised the manuscript. NC and KG undertook data collection and revised the manuscript. AG contributed to development of the analysis plan and revised the manuscript. All authors have approved the final version of the manuscript.

\section{Author information}

LMW holds a PhD and is Professor of Psychiatry and Behavioral Sciences at Stanford University and Director of the Panlab at Stanford and the VA Palo Alto MIRECC, AG holds a PhD and is a Postdoctoral Fellow in the Panlab, Psychiatry and Behavioral Sciences at Stanford University, NC holds a BSC and is a clinical research coordinator in the PanLab Psychiatry and Behaviora Sciences at Stanford University, KG holds a BSC and is a clinical research coordinator in the PanLab Psychiatry and Behavioral Sciences at Stanford University, NH is the Research Director of the Gronowski Center of Palo Alto University. TS is a M.D., Ph.D., and is Professor of Stanford University in the School of Medicine, Department of Psychiatry and Behavioral Sciences. She is a staff physician in the VA Palo Alto Health Care System and Director of the Bipolar and Depression Research Program there, as well as Director of the VA CSP Network of Dedicated Enrollment Sites.

\section{Acknowledgements}

This study is funded by the National Institute of Mental Health in response to an RDoC RFA and the grant award identification is R01MH101496. The authors thank the clinical directors and student therapists of the Gronowski Center for their partnership and valued time.

\section{Data sharing statement}

All data resulting from this $\mathrm{NIH}$-funded research involving human subjects are expected to be submitted to the Research Domain Criteria database (RDoC db), along with appropriate supporting documentation to enable efficient use of the data. The goal of this data sharing policy is to facilitate RDoC research and foster collaboration by giving the broader research community access to publicly available high-quality data.

\section{Author details}

'Department of Psychiatry and Behavioral Sciences, Stanford University School of Medicine, Stanford, CA 94305, USA. ${ }^{2}$ Sierra-Pacific Mental Illness
Research, Education, and Clinical Center (MIRECC) Veterans Affairs Palo Alto Health Care System, Palo Alto, CA 94304, USA. ${ }^{3}$ Psychology, Palo Alto University, 1791 Arastradero Road, Palo Alto, CA 94304, USA. ${ }^{4}$ Veterans Affairs Palo Alto Health Care System, Palo Alto, CA 94304, USA.

Received: 30 January 2016 Accepted: 8 March 2016

Published online: 15 March 2016

\section{References}

1. Baxter AJ, Scott KM, Vos T, Whiteford HA. Global prevalence of anxiety disorders: a systematic review and meta-regression. Psychol Med. 2013;43(5):897-910.

2. Ferrari AJ, Somerville AJ, Baxter AJ, Norman R, Patten SB, Vos T, Whiteford HA. Global variation in the prevalence and incidence of major depressive disorder: a systematic review of the epidemiological literature. Psychol Med. 2013;43(3):471-81.

3. Whiteford HA, Degenhardt L, Rehm J, Baxter AJ, Ferrari AJ, Erskine HE, Charlson FJ, Norman RE, Flaxman AD, Johns N, et al. Global burden of disease attributable to mental and substance use disorders: findings from the Global Burden of Disease Study 2010. Lancet. 2013;382(9904):1575-86.

4. American Psychiatric Association. Diagnostic and Statistical Manual of Mental Disorders: DSM-5. 5th ed. Washington, D.C: American Psychiatric Association; 2013

5. Somers JM, Goldner EM, Waraich P, Hsu L. Prevalence and incidence studies of anxiety disorders: a systematic review of the literature. Can J Psychiatry. 2006;51(2):100-13.

6. Substance Abuse and Mental Health Services Administration. Results from the 2012 National Survey on Drug Use and Health: Mental Health Findings, NSDUH Series H-47, HHS Publication No. (SMA) 13-4805. Rockville, MD: Substance Abuse and Mental Health Services Administration, 2013.

7. Weissman MM, Bland RC, Canino GJ, Faravelli C, Greenwald S, Hwu HG, Joyce PR, Karam EG, Lee CK, Lellouch J, et al. The cross-national epidemiology of panic disorder. Arch Gen Psychiatry. 1997;54(4):305-9.

8. Grohol J. Top 25 Psychiatric Medication Prescriptions for 2013. Psych Central; 2015. http://psychcentral.com/lib/top-25-psychiatric-medicationprescriptions-for-2013/.

9. Cuthbert BN, Insel TR. Toward the future of psychiatric diagnosis: the seven pillars of RDoC. BMC Med. 2013;11:126.

10. Insel T, Cuthbert B, Garvey M, Heinssen R, Pine DS, Quinn K, Sanislow C, Wang P. Research domain criteria (RDoC): toward a new classification framework for research on mental disorders. Am J Psychiatry. 2010;167(7):748-51.

11. Williams L. Precision psychiatry: a neural circuit taxonomy for depression and anxiety. Lancet. 2016.

12. Woody ML, Gibb BE. Integrating NIMH Research Domain Criteria (RDoC) into depression research. Curr Opin Psychol. 2015;4:6-12.

13. Kober H, Barrett LF, Joseph J, Bliss-Moreau E, Lindquist K, Wager TD. Functional grouping and cortical-subcortical interactions in emotion: a meta-analysis of neuroimaging studies. Neuroimage. 2008;42(2):998-1031.

14. Robinson OJ, Krimsky M, Lieberman L, Allen P, Vytal K, Grillon C. The dorsal medial prefrontal (anterior cingulate) cortex-amygdala aversive amplification circuit in unmedicated generalised and social anxiety disorders: an observational study. Lancet Psychiatry. 2014;1(4):294-302.

15. Etkin A, Prater KE, Hoeft F, Menon V, Schatzberg AF. Failure of anterior cingulate activation and connectivity with the amygdala during implicit regulation of emotional processing in generalized anxiety disorder. Am J Psychiatry. 2010;167(5):545-54.

16. Williams LM, Das P, Liddell BJ, Kemp AH, Rennie CJ, Gordon E. Mode of functional connectivity in amygdala pathways dissociates level of awareness for signals of fear. J Neurosci. 2006;26(36):9264-71.

17. Niendam TA, Laird AR, Ray KL, Dean YM, Glahn DC, Carter CS. Meta-analytic evidence for a superordinate cognitive control network subserving diverse executive functions. Cogn Affect Behav Neurosci. 2012;12(2):241-68.

18. Cole MW, Schneider W. The cognitive control network: integrated cortical regions with dissociable functions. Neuroimage. 2007;37(1):343-60.

19. Roalf DR, Ruparel K, Gur RE, Bilker W, Gerraty R, Elliott MA, Gallagher RS, Almasy L, Pogue-Geile MF, Prasad K. Neuroimaging predictors of cognitive performance across a standardized neurocognitive battery. Neuropsychology. 2014;28(2):161-76.

20. Jaworska N, Yang XR, Knott $V$, MacQueen G. A review of $\mathrm{fMRI}$ studies during visual emotive processing in major depressive disorder. World J Biol Psychiatry. 2015;16(7):448-71. 
21. Fonzo GA, Ramsawh HJ, Flagan TM, Sullivan SG, Letamendi A, Simmons AN, Paulus MP, Stein MB. Common and disorder-specific neural responses to emotional faces in generalised anxiety, social anxiety and panic disorders. $\mathrm{Br}$ J Psychiatry. 2015;206(3):206-15.

22. Stein MB, Goldin PR, Sareen J, Zorrilla LT, Brown GG. Increased amygdala activation to angry and contemptuous faces in generalized social phobia. Arch Gen Psychiatry. 2002;59(11):1027-34.

23. Phan KL, Fitzgerald DA, Nathan PJ, Tancer ME. Association between amygdala hyperactivity to harsh faces and severity of social anxiety in generalized social phobia. Biol Psychiatry. 2006;59(5):424-9.

24. Killgore WD, Britton JC, Schwab ZJ, Price LM, Weiner MR, Gold AL,Rosso IM, Simon NM, Pollack MH, Rauch SL. Cortico-limbic responses to masked affective faces across ptsd, panic disorder, and specific phobia. Depress Anxiety. 2014;31(2):150-9.

25. Fu CH, Williams SC, Cleare AJ, Brammer MJ, Walsh ND, Kim J, Andrew CM, Pich EM, Williams PM, Reed LJ, et al. Attenuation of the neural response to sad faces in major depression by antidepressant treatment: a prospective, event-related functional magnetic resonance imaging study. Arch Gen Psychiatry. 2004;61(9):877-89.

26. Victor TA, Furey ML, Fromm SJ, Ohman A, Drevets WC. Relationship between amygdala responses to masked faces and mood state and treatment in majo depressive disorder. Arch Gen Psychiatry. 2010;67(11):1128-38.

27. Williams LM, Korgaonkar MS, Song YC, Paton R, Eagles S, Goldstein-Piekarski A, Grieve SM, Harris AW, Usherwood T, Etkin A. Amygdala Reactivity to Emotional Faces in the Prediction of General and Medication-Specific Responses to Antidepressant Treatment in the Randomized iSPOT-D Trial. Neuropsychopharmacology 2015, 40(10):2398-2408.

28. Suslow T, Konrad C, Kugel H, Rumstadt D, Zwitserlood P, Schoning S, Ohrmann P, Bauer J, Pyka M, Kersting A, et al. Automatic mood-congruent amygdala responses to masked facial expressions in major depression. Biol Psychiatry. 2010;67(2):155-60

29. Arnone D, McKie S, Elliott R, Thomas EJ, Downey D, Juhasz G, Williams SR, Deakin JF, Anderson IM. Increased amygdala responses to sad but not fearful faces in major depression: relation to mood state and pharmacological treatment. Am J Psychiatry. 2012;169(8):841-50.

30. Blair KS, Geraci M, Smith BW, Hollon N, DeVido J, Otero M, Blair JR, Pine DS. Reduced dorsal anterior cingulate cortical activity during emotional regulation and top-down attentional control in generalized social phobia, generalized anxiety disorder, and comorbid generalized social phobia/ generalized anxiety disorder. Biol Psychiatry. 2012;72(6):476-82.

31. Matthews SC, Strigo IA, Simmons AN, Yang TT, Paulus MP. Decreased functional coupling of the amygdala and supragenual cingulate is related to increased depression in unmedicated individuals with current major depressive disorder. J Affect Disord. 2008;111(1):13-20.

32. Prater KE, Hosanagar A, Klumpp H, Angstadt M, Phan KL. Aberrant amygdalafrontal cortex connectivity during perception of fearful faces and at rest in generalized social anxiety disorder. Depress Anxiety. 2013;30(3):234-41.

33. Rose EJ, Simonotto E, Ebmeier KP. Limbic over-activity in depression during preserved performance on the n-back task. Neuroimage. 2006;29(1):203-15.

34. Matsuo K, Glahn DC, Peluso MA, Hatch JP, Monkul ES, Najt P, Sanches M, Zamarripa F, Li J, Lancaster JL, et al. Prefrontal hyperactivation during working memory task in untreated individuals with major depressive disorder. Mol Psychiatry. 2007;12(2):158-66.

35. Siegle GJ, Thompson W, Carter CS, Steinhauer SR, Thase ME. Increased amygdala and decreased dorsolateral prefrontal BOLD responses in unipolar depression: related and independent features. Biol Psychiatry. 2007;61(2):198-209.

36. Elliott R, Sahakian BJ, Herrod JJ, Robbins TW, Paykel ES. Abnormal response to negative feedback in unipolar depression: evidence for a diagnosis specific impairment. J Neurol Neurosurg Psychiatry. 1997;63(1):74-82.

37. Korgaonkar MS, Grieve SM, Etkin A, Koslow SH, Williams LM. Using standardized fMRI protocols to identify patterns of prefrontal circuit dysregulation that are common and specific to cognitive and emotional tasks in major depressive disorder: first wave results from the iSPOT-D study. Neuropsychopharmacology. 2013;38(5):863-71.

38. Koric L, Volle E, Seassau M, Bernard FA, Mancini J, Dubois B, Pelissolo A, Levy R. How cognitive performance-induced stress can influence right VLPFC activation: an fMRI study in healthy subjects and in patients with social phobia. Hum Brain Mapp. 2012;33(8):1973-86.

39. Aizenstein HJ, Butters MA, Wu M, Mazurkewicz LM, Stenger VA, Gianaros PJ, Becker JT, Reynolds CF 3rd, Carter CS. Altered functioning of the executive control circuit in late-life depression: episodic and persistent phenomena. Am J Geriatr Psychiatry. 2009;17(1):30-42.

40. Risbrough V. Behavioral correlates of anxiety. Curr Top Behav Neurosci. 2010;2:205-28.

41. Craske MG, Rauch SL, Ursano R, Prenoveau J, Pine DS, Zinbarg RE. What is an anxiety disorder? Depress Anxiety. 2009;26(12):1066-85.

42. den Hollander-Gijsman ME, Wardenaar KJ, de Beurs E, van der Wee NJ, Mooijaart A, van Buuren S, Zitman FG. Distinguishing symptom dimensions of depression and anxiety: an integrative approach. J Affect Disord. 2012;136(3):693-701.

43. Beaudreau SA, O'Hara R. Late-life anxiety and cognitive impairment: a review. Am J Geriatr Psychiatry. 2008;16(10):790-803.

44. Sheehan DV, Lecrubier $Y$, Sheehan $\mathrm{KH}$, Amorim P, Janavs J, Weiller $\mathrm{E}$, Hergueta T, Baker R, Dunbar GC. The Mini-International Neuropsychiatric Interview (M.I.N.I.): the development and validation of a structured diagnostic psychiatric interview for DSM-IV and ICD-10. J Clin Psychiatry. 1998;59 Suppl 20:22-33. quiz 34-57.

45. Korgaonkar MS, Cooper NJ, Williams LM, Grieve SM. Mapping inter-regional connectivity of the entire cortex to characterize major depressive disorder: a whole-brain diffusion tensor imaging tractography study. Neuroreport. 2012;23(9):566-71.

46. Liddell BJ, Brown KJ, Kemp AH, Barton MJ, Das P, Peduto A, Gordon E, Williams LM. A direct brainstem-amygdala-cortical 'alarm' system for subliminal signals of fear. Neuroimage. 2005;24(1):235-43.

47. Williams LM, Liddell BJ, Kemp AH, Bryant RA, Meares RA, Peduto AS, Gordon E. Amygdala-prefrontal dissociation of subliminal and supraliminal fear. Hum Brain Mapp. 2006;27(8):652-61.

48. Williams LM, Liddell BJ, Rathjen J, Brown KJ, Gray J, Phillips M, Young A, Gordon E. Mapping the time course of nonconscious and conscious perception of fear: an integration of central and peripheral measures. Hum Brain Mapp. 2004;21(2):64-74.

49. Wild B, Erb M, Bartels M. Are emotions contagious? Evoked emotions while viewing emotionally expressive faces: quality, quantity, time course and gender differences. Psychiatry Res. 2001.

50. Costafreda SG, Brammer MJ, David AS, Fu CH. Predictors of amygdala activation during the processing of emotional stimuli: a meta-analysis of 385 PET and fMRI studies. Brain Res Rev. 2008;58(1):57-70.

51. Etkin A, Egner T, Peraza DM, Kandel ER, Hirsch J. Resolving emotional conflict: a role for the rostral anterior cingulate cortex in modulating activity in the amygdala. Neuron. 2006;51(6):871-82.

52. Ekman P, Friesen WV. Pictures of Facial Affect. Consulting Psychologists Press; 1976.

53. Falconer E, Allen A, Felmingham KL, Williams LM, Bryant RA. Inhibitory neural activity predicts response to cognitive-behavioral therapy for posttraumatic stress disorder. J Clin Psychiatry. 2013;74(9):895-901.

54. Falconer E, Bryant R, Felmingham KL, Kemp AH, Gordon E, Peduto A, Olivieri $G$, Williams LM. The neural networks of inhibitory control in posttraumatic stress disorder. J Psychiatry Neurosci. 2008;33(5):413-22.

55. Ashburner J, Friston KJ. Unified segmentation. Neuroimage. 2005;26(3):839-51.

56. Patenaude B, Smith SM, Kennedy DN, Jenkinson M. A Bayesian model of shape and appearance for subcortical brain segmentation. Neuroimage. 2011;56(3):907-22

57. Watters AJ, Williams LM. Negative biases and risk for depression; integrating self-report and emotion task markers. Depress Anxiety. 2011;28(8):703-18.

58. Egner T, Etkin A, Gale S, Hirsch J. Dissociable neural systems resolve conflict from emotional versus nonemotional distracters. Cereb Cortex. 2008;18(6):1475-84.

59. Mathersul D, Palmer DM, Gur RC, Gur RE, Cooper N, Gordon E, Williams LM. Explicit identification and implicit recognition of facial emotions: II. Core domains and relationships with general cognition. J Clin Exp Neuropsychol. 2009;31(3):278-91.

60. Williams LM, Simms E, Clark CR, Paul RH, Rowe D, Gordon E. The test-retest reliability of a standardized neurocognitive and neurophysiological test battery: "neuromarker". Int J Neurosci. 2005;115(12):1605-30.

61. Clark CR, Paul RH, Williams LM, Arns M, Fallahpour K, Handmer C, Gordon E. Standardized assessment of cognitive functioning during development and aging using an automated touchscreen battery. Arch Clin Neuropsychol. 2006;21(5):449-67.

62. Paul RH, Clark CR, Lawrence J, Goldberg E, Williams LM, Cooper N, Cohen RA, Brickman AM, Gordon E. Age-dependent change in executive function and gamma $40 \mathrm{~Hz}$ phase synchrony. J Integr Neurosci. 2005;4(1):63-76.

63. Paul RH, Gunstad J, Cooper N, Williams LM, Clark CR, Cohen RA, Lawrence JJ, Gordon E. Cross-cultural assessment of neuropsychological performance 
and electrical brain function measures: additional validation of an international brain database. Int J Neurosci. 2007;117(4):549-68.

64. Williams LM, Mathersul D, Palmer DM, Gur RC, Gur RE, Gordon E. Explicit identification and implicit recognition of facial emotions: I. Age effects in males and females across 10 decades. J Clin Exp Neuropsychol. 2009:31(3):257-77.

65. Liddell BJ, Paul RH, Arns M, Gordon N, Kukla M, Rowe D, Cooper N, Moyle J, Williams LM. Rates of decline distinguish Alzheimer's disease and mild cognitive impairment relative to normal aging: integrating cognition and brain function. J Integr Neurosci. 2007;6(1):141-74.

66. Hatch A, Madden S, Kohn M, Clarke S, Touyz S, Williams LM. Anorexia nervosa: towards an integrative neuroscience model. Eur Eat Disord Rev. 2010;18(3):165-79.

67. Williams LM, Hermens DF, Thein T, Clark CR, Cooper NJ, Clarke SD, Lamb C, Gordon E, Kohn MR. Using brain-based cognitive measures to support clinical decisions in ADHD. Pediatr Neurol. 2010;42(2):118-26.

68. Williams LM, Whitford TJ, Flynn G, Wong W, Liddell BJ, Silverstein S, Galletly C, Harris AW, Gordon E. General and social cognition in first episode schizophrenia: identification of separable factors and prediction of functional outcome using the IntegNeuro test battery. Schizophr Res. 2008;99(1-3):182-91.

69. Beck AT, Epstein N, Brown G, Steer RA. An inventory for measuring clinical anxiety: psychometric properties. J Consult Clin Psychol. 1988;56(6):893-7.

70. Meyer TJ, Miller ML, Metzger RL, Borkovec TD. Development and validation of the Penn State Worry Questionnaire. Behav Res Ther. 1990;28(6):487-95.

71. Beck AT, Ward CH, Mendelson M, Mock J, Erbaugh J. An inventory for measuring depression. Arch Gen Psychiatry. 1961;4:561-71.

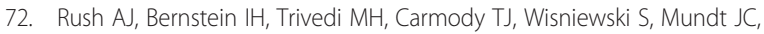
Shores-Wilson K, Biggs MM, Woo A, Nierenberg AA, et al. An evaluation of the quick inventory of depressive symptomatology and the hamilton rating scale for depression: a sequenced treatment alternatives to relieve depression trial report. Biol Psychiatry. 2006;59(6):493-501.

73. Lovibond L. Manual for the depression anxiety stress scales. 2nd ed. Sydney: Psychology Foundation of Australia, 1996; 1995.

74. Watson D, Weber K, Assenheimer JS, Clark LA, Strauss ME, McCormick RA. Testing a tripartite model: I. Evaluating the convergent and discriminant validity of anxiety and depression symptom scales. J Abnorm Psychol. 1995; 104(1):3-14.

75. Blanchard EB, Jones-Alexander J, Buckley TC, Forneris CA. Psychometric properties of the PTSD Checklist (PCL). Behav Res Ther. 1996;34(8):669-73.

76. Patton $\mathrm{JH}$, Stanford MS, Barratt ES. Factor structure of the Barratt impulsiveness scale. J Clin Psychol. 1995;51(6):768-74

77. Heatherton TF, Kozlowski LT, Frecker RC, Fagerstrom KO. The Fagerstrom Test for Nicotine Dependence: a revision of the Fagerstrom Tolerance Questionnaire. Br J Addict. 1991;86(9):1119-27.

78. Adamson SJ, Kay-Lambkin FJ, Baker AL, Lewin TJ, Thornton L, Kelly BJ, Sellman JD. An improved brief measure of cannabis misuse: the Cannabis Use Disorders Identification Test-Revised (CUDIT-R). Drug Alcohol Depend. 2010;110(1-2):137-43.

79. The National Institute on Drug Abuse (NIDA). Screening for Drug Use in General Medical Settings: The NIDA Quick Screen. 2009.

80. Chu DA, Williams LM, Harris AW, Bryant RA, Gatt JM. Early life trauma predicts self-reported levels of depressive and anxiety symptoms in nonclinical community adults: relative contributions of early life stressor types and adult trauma exposure. J Psychiatr Res. 2013;47(1):23-32.

81. Carver CS, Scheier MF, Weintraub JK. Assessing coping strategies: a theoretically based approach. J Pers Soc Psychol. 1989;56(2):267-83.

82. Gross JJ, John OP. Individual differences in two emotion regulation processes: implications for affect, relationships, and well-being. J Pers Soc Psychol. 2003;85(2):348-62.

83. Goldman HH, Skodol AE, Lave TR. Revising axis $V$ for DSM-IV: a review of measures of social functioning. Am J Psychiatry. 1992;149(9):1148-56.

84. Development of the World Health Organization WHOQOL-BREF quality of life assessment. The WHOQOL Group. Psychol Med. 1998;28:551-8.

85. Diener E, Emmons RA, Larsen RJ, Griffin S. The satisfaction with life scale. J Pers Assess. 1985;49(1):71-5

86. Kessler RC, Barber C, Beck A, Berglund P, Cleary PD, McKenas D, Pronk N, Simon G, Stang P, Ustun TB, et al. The World Health Organization Health and Work Performance Questionnaire (HPQ). J Occup Environ Med. 2003:45(2):156-74.
87. Carvalho CM, Chang J, Lucas JE, Nevins JR, Wang Q, West M. High-dimensional sparse factor modeling: applications in gene expression genomics. J Am Stat Assoc. 2008;103(484):1438-56.

88. Jensen FV, Nielsen TD. Bayesian Networks and Decision Graphs. 2nd ed. New York: Springer; 2007.

89. Zhu J, Hastie T. Classification of gene microarrays by penalized logistic regression. Biostatistics. 2004;5(3):427-43.

90. Cohen J, Cohen P, West SG, Aiken LS. Applied multiple regression/ correlation analysis for the behavioral sciences. 3rd ed. Mahwah: Lawrence Erlbaum Associates; 2003.

\section{Submit your next manuscript to BioMed Central and we will help you at every step:}

- We accept pre-submission inquiries

- Our selector tool helps you to find the most relevant journal

- We provide round the clock customer support

- Convenient online submission

- Thorough peer review

- Inclusion in PubMed and all major indexing services

- Maximum visibility for your research

Submit your manuscript at www.biomedcentral.com/submit
Biomed Central 